\title{
ALL AUTOHOMEOMORPHISMS OF CONNECTED MENGER MANIFOLDS ARE STABLE
}

\author{
KATSURO SAKAI
}

(Communicated by James E. West)

\begin{abstract}
Generalizing the result of Chigogidze (1991), we prove that all autohomeomorphisms of connected Menger manifolds are stable (in the sense of Brown and Gluck).
\end{abstract}

A manifold modeled on the $(n+1)$-dimensional universal Menger compactum $\mu^{n+1}$ is called a $\mu^{n+1}$-manifold. In $\left[\mathrm{C}_{1}\right]$, Chigogidze proved that an autohomeomorphism $h$ of a $\mu^{n+1}$-manifold is stable (in the sense of Brown and Gluck [BG]) if $h$ is properly $n$-homotopic to id. Here we show that all autohomeomorphisms of connected $\mu^{n+1}$-manifolds are stable. Indeed the following can be proved:

Theorem. Every autohomeomorphism $h$ of a connected $\mu^{n+1}$-manifold $M$ can be written as the composition of two homeomorphisms, each of which is identity on some nonempty open set.

The connectedness is necessary. In fact, let $M=M_{1} \oplus M_{2}$, where $M_{1} \approx$ $M_{2}$ are connected. ${ }^{1}$ Then any autohomeomorphism $h$ of $M$ is unstable if $h\left(M_{1}\right)=M_{2}$.

A pair $(W, \delta W)$ of compacta in $M$ is called a clean $\mu^{n+1}$-pair if

(1) $W \approx \delta W \approx \mu^{n+1}$,

(2) $(M \backslash W) \cup \delta W$ is a $\mu^{n+1}$-manifold,

(3) $\delta W$ is a $Z$-set in both $W$ and $(M \backslash W) \cup \delta W$, and

(4) $W \backslash \delta W$ is open in $M$ (i.e., $\left.\operatorname{bd}_{M} W \subset \delta W\right) .^{2}$

Lemma. Let $(W, \delta W)$ be a clean $\mu^{n+1}$-pair in a $\mu^{n+1}$-manifold $M$. Then $(M \backslash W) \cup \delta W \approx M$.

Proof. First note that the inclusion $j:(M \backslash W) \cup \delta W \subset M$ induces a homeomorphism between the spaces of ends, whence $j$ induces an isomorphism of homotopy groups of ends. By [Be, §6, Theorem], it suffices to show that $j$ induces an isomorphism of homotopy groups of $\operatorname{dim} \leqslant n$.

Received by the editors September 9, 1993.

1991 Mathematics Subject Classification. Primary 57N99, 54F15, 57N20.

Key words and phrases. Autohomeomorphism, Menger manifold, stable.

${ }^{1}$ Here " $\approx$ " means "is homeomorphic to".

${ }^{2}$ The existence of such a pair is guaranteed by [IS, Lemma 1] (cf. [ $\left.C_{2}\right]$ ). 
Epi: For each map $f: \mathbf{S}^{i} \rightarrow M(i \leqslant n)$ of the $i$-sphere, let

$$
A=\operatorname{cl} f^{-1}(W \backslash \delta W) \text { and } B=\operatorname{bd} A .
$$

By [Hu, Chapter V, Theorem 10.1], $f \mid B$ extends to a map $g^{\prime}: A \rightarrow \delta W$ and there is a homotopy $h^{\prime}: A \times \mathbf{I} \rightarrow W$ such that $h_{0}^{\prime}=f \mid A, h_{1}^{\prime}=g^{\prime}$, and $h_{t}^{\prime}|B=f| B$ for each $t \in \mathbf{I}$. We can extend $g^{\prime}$ and $h^{\prime}$ to $g: \mathbf{S}^{i} \rightarrow(M \backslash W) \cup \delta W$ and $h: \mathbf{S}^{i} \times \mathbf{I} \rightarrow M$ by $g\left|\mathbf{S}^{i} \backslash A=h_{t}\right| \mathbf{S}^{i} \backslash A=f \mid \mathbf{S}^{i} \backslash A$. Then $h_{0}=f$ and $h_{1}=g$. This means that $j$ induces an epimorphism of homotopy groups of $\operatorname{dim} \leqslant n$.

Mono: Suppose that a map $g: \mathbf{S}^{i} \rightarrow(M \backslash W) \cup \delta W(i \leqslant n)$ extends to a map $f: \mathbf{B}^{i+1} \rightarrow M$ of the $(i+1)$-ball. Let $C=\operatorname{cl} f^{-1}(W \backslash \delta W)$ and $D=\operatorname{bd} C$. Similarly as above, $f \mid D$ extends to a map $h^{\prime}: C \rightarrow \delta W$. We can extend $h^{\prime}$ to $h: \mathbf{B}^{i+1} \rightarrow(M \backslash W) \cup \delta W$ by $h\left|\mathbf{B}^{i+1} \backslash C=f\right| \mathbf{B}^{i+1} \backslash C$. Since $\mathbf{S}^{i} \subset\left(\mathbf{B}^{i+1} \backslash C\right) \cup D$, $h\left|\mathbf{S}^{i}=f\right| \mathbf{S}^{i}=g$. This implies that $j$ induces a monomorphism of homotopy groups of $\operatorname{dim} \leqslant n$.

Proof of Theorem. Let $\left(W_{0}, \delta W_{0}\right)$ be a clean $\mu^{n+1}$-pair in $M$ and $a \in M$. Since $M$ is connected, by the $Z$-set Unknotting Theorem [Be, $\S 6]$, we have an autohomeomorphism $f$ of $M$ such that $f(a), f(h(a)) \in W_{0} \backslash \delta W_{0}$. Then $(W, \delta W)=\left(f^{-1}\left(W_{0}\right), f^{-1}\left(\delta W_{0}\right)\right)$ is a clean $\mu^{n+1}$-pair and $a, h(a) \in W \backslash \delta W$. By [IS, Lemma 1], we can choose a clean $\mu^{n+1}$-pair $(V, \delta V)$ so that $a \in V \backslash \delta V$ and $V \cup h(V) \subset W \backslash \delta W$. Note that $(h(V), h(\delta V))$ is also a clean $\mu^{n+1}$-pair. By Lemma, $N_{1}=(W \backslash V) \cup \delta V \approx \mu^{n+1}$ and $N_{2}=(W \backslash h(V)) \cup h(\delta V) \approx$ $\mu^{n+1}$. Observe that $\delta W$ and $\delta V$ are disjoint $Z$-sets in $N_{1}$, and $\delta W$ and $h(\delta V)$ are disjoint $Z$-sets in $N_{2}$. By using the $Z$-set Unknotting Theorem [Be, 3.1.5], we have a homeomorphism $g: N_{1} \rightarrow N_{2}$ such that $g \mid \delta W=$ id and $g|\delta V=h| \delta V$, which can be extended to a homeomorphism $h_{1}: M \rightarrow M$ by $h_{1} \mid M \backslash W=$ id and $h_{1}|V=h| V$. Then $h=h_{2} \circ h_{1}$, where $h_{2}=h_{\circ} h_{1}^{-1}: M \rightarrow M$ is a homeomorphism such that $h_{2} \mid V=\mathrm{id}$.

Remark. In [Wo], [CM], and [Mc], the stability of all autohomeomorphisms is shown for connected manifolds modeled on the Hilbert cube $Q$ and a normed linear space $E \approx E^{\omega}$ or $\approx E_{f}^{\omega}$. Our approach is valid for these manifolds and also for manifolds modeled on the direct limits $\mathbb{R}^{\infty}=\operatorname{dir} \lim \mathbb{R}^{n}$ and $Q^{\infty}=\operatorname{dir} \lim Q^{n}$. For required techniques, refer to [Ch], [BP], and [S $\mathrm{S}_{1,2}$.

\section{REFERENCES}

[BP] C. Bessaga and A. Pełczyński, Selected topics in infinite-dimensional topology, Monograf. Mat., vol. 58, Polish Sci. Publ., Warsaw, 1975.

[Be] M. Bestvina, Characterizing k-dimensional universal Menger compacta, Mem. Amer. Math. Soc. (no.380) 71 (1988).

[BG] M. Brown and H. Gluck, Stable structures on manifolds: I, II, III, Ann. of Math. (2) 79 (1964), 1-58.

[Ch] T. A. Chapman, Lectures on Hilbert cube manifolds, CBMS Regional Conf. Ser. in Math., vol. 28, Amer. Math. Soc., Providence, RI, 1976.

$\left[\mathrm{C}_{1}\right]$ A. Chigogidze, Autohomeomorphisms of the universal Menger compacta are stable, Soobshch Akad. Nauk Gruz. SSR 142 (3) (1991), 477-479.

$\left[\mathrm{C}_{2}\right]$, Finding boundaries for Menger manifolds, Proc. Amer. Math. Soc. 121 (1994), 631-640. 
[CM] D. Curtis and R. A. McCoy, Stable homeomorphisms on infinite-dimensional normed linear spaces, Proc. Amer. Math. Soc. 28 (1971), 496-500.

[Hu] S.-T. Hu, Theory of retracts, Wayne State Univ. Press, Detroit, MI, 1965.

[IS] Y. Iwamoto and K. Sakai, A mapping theorem for Q-manifolds and $\mu^{n+1}$-manifolds, preprint.

[Mc] R. A. McCoy, Groups of homeomorphisms of normed linear spaces, Pacific J. Math. 39 (1971), 735-743.

[S $\left.\mathrm{S}_{1}\right] \quad \mathrm{K}$. Sakai, On $\mathbb{R}^{\infty}$-manifolds and $Q^{\infty}$-manifolds, Topology Appl. 18 (1984), 69-80.

[S $\left.\mathrm{S}_{2}\right] \quad$, On $\mathbb{R}^{\infty}$-manifolds and $Q^{\infty}$-manifolds, II: Infinite deficiency, Tsukuba J. Math. 8 (1984), 101-118.

[Wo] R. Y. T. Wong, A note on stable homeomorphisms of infinite-dimensional manifolds, Proc. Amer. Math. Soc. 28 (1971), 271-272.

Institute of Mathematics, University of Tsukuba, Tsukuba-city 305, JaPan

E-mail address: sakaiktr@sakura.cc.tsukuba.ac.jp 